\title{
方向 EMD分解与其在纹理分割中的应用
}

\author{
刘忠轩 彭思龙 \\ (中国科学院自动化研究所 国家专用集成电路中心, 北京 100080)
}

摘要提出了方向 EMD(Directional Empirical Mode Decomposition, 简写为 DEMD)的定义和框架并用来进行纹理分割. EMD是由Huang等人提出的新的时频 分析工具. 这种工具使用篮法对信号分解, 再对成分的瞬时频率进行分析. 虽然 已有文章讨论将一维EMD推广到二维情况, 但有两个方面还没有考虑: 一方面 是从二维EMD中提取特征, 另一方面是考虑图像固有的方向性在二维EMD分解 中的作用. 文中考虑到这两方面, 提出了DEMD的方法. 这种方法在分解框架中 考虑了图像的方向性, 并从分解的每个成分中对每个点提出三个特征以进行图 像处理. 使用DEMD分解提取的特征进行纹理分割的实验说明了这种方法对于纹 理处理的有效性. 此外, 给出了关于DEMD区分纹理在视觉上的解释.

关键词 EMD(Empirical Mode Decomposition) 方向 EMD IMF(Intrinsic Mode Function) 多尺度 纹理分割

图像处理现在已经成为一门有着系统理论和广泛应用的学科, 其应用领域 包括视频影像处理、医学图像处理、遥感图像处理以及最近得到广泛关注的生物 特征识别技术和视觉监控技术等. 多尺度特性是自然图像的主要特性之一，因而 图像处理中已提出一系列体现图像多尺度特性的方法, 如：基于扩散方程的多尺

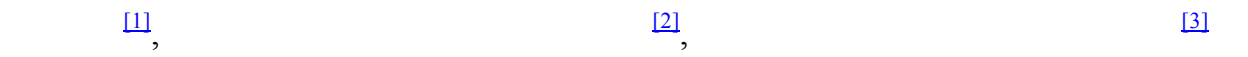
及近十年来得到广泛研究的小波多分辨分析框架 ${ }^{[4]}$. 二维经验模式分解 (Bidimensional Empirical Mode Decomposition, BEMD) ${ }^{[5]}$ 是最近提出的新的图像 多尺度分析方法, 其与传统多尺度分析技术区别主要在于: 首先, 极值点距离被 引入进行局部尺度的确定从而使分解具有自适应和完全数据驱动的特性; 其次, 每一成分的提取都使用迭代计算方法, 并使用某种准则确定迭代的终止. 由于这 些原因, BEMD在自适应的提取图像符合视觉感知的成分上有其独特的优势. 但

2004-08-05 收稿, 2004-11-30 收修改稿 
作为一种新兴的技术, BEMD存在一系列难题: 第一, 方法缺乏理论基础; 第二, 很少有关于特征提取的讨论和具体应用的尝试; 第三, 文献[5]中使用的径向基函 数提取包络的方法在计算量上和存储量上开销太大. 本文就是针对后两个难题, 提出了一种新的BEMD框架, 称为方向EMD分解(Directional EMD, DEMD), 具有 计算量较小的优点, 并且用所提出的特征进行纹理分割.

纹理分割是自然图像感知编组的主要问题之一. 关于这个问题已经提出了 大量的方法, 这些方法可以分为四类: 基于统计的方法如利用自回归模型 [6]; 基 于结构的方法如基于Voronoi 图的方法 ${ }^{[7]}$; 基于模型的方法如考虑图像的分形模 型 ${ }^{[8]}$; 基于空间频率域分析/多尺度滤波的方法 ${ }^{[9]}$ 如基于小波、Gabor滤波器的方法. 虽然这些方法都有其各自的优点, 但只有第四种方法反映了人视觉系统的空频 局部特性和多尺度特性，关于这类方法最近又有了一系列进展. Lu等 ${ }^{[10]}$ 提出了基 于小波变换的非监督纹理分割方法, 这种方法有两个问题: 一是所抽取的特征只 能反映图像在单一函数经过平移和伸缩所构成函数上的能量; 二是需要对小波 函数、分解层数和一些参数进行选取. 基于Gabor滤波器的纹理特征是MPEG-7 中 纹理分类所使用的主要特征之一 ${ }^{[11]}$, 近年来获得广泛注意. 但这些特征有几余的 问题. Gabor滤波器特征的冗余性可以用优化滤波器方法 ${ }^{[12]}$ 部分的解决. 但 Randen等人 ${ }^{[13]}$ 证明在不严重减少分割性能的前提下减少滤波器数目是不可能的.

本文提出将DEMD这种新的多尺度分析方法用来进行纹理分割, 这种方法 可以归于空频分析的范畴, 因为其将图像分解成分的瞬时频率作为特征. 在此之 前, 一方面, Gabor滤波器和小波方法通过提取具有空间、频率/尺度局部性的滤波 器输出的包络作为纹理分割的特征, 频率由于已为滤波器频率事先确定而未被用 来作为特征; 另一方面, 将原信号定义的瞬时频率作为特征存在一些问题 ${ }^{[14]}$, 例如: 多成分信号在单一时间只具有单一频率, 频率可能有负值等. Huang等人 ${ }^{[15]}$ 提出的 EMD (empirical mode decomposition)方法通过先使用“篮”法对信号进行分解再利 用Hilbert变换定义每个成分的复信号和瞬时频率而在一维上解决了这个问题. 对 于二维信号(图像)的EMD分析方法(二维EMD)也有讨论, 但只是对图像每行进行 EMD处理 ${ }^{[16,17]}$, 或仅对图像利用径向基函数进行分解 ${ }^{[5]}$ 而没有提出有效的图像 特征, 图像的方向特性也没有被考虑. 有鉴于此, 本文提出考虑方向性的类似于 张量积型分解一DEMD分解，并从中提取特征进行纹理分割. 另外，本文应用纹 理的Wold分解 ${ }^{[18]}$ 进行DEMD分解方向的确定. 与已被录取的会议文 ${ }^{\text {章 }}{ }^{[19]}$ 不同, 本文考虑了DEMD分解的视觉意义, 进一步作了方向确定的讨论和实验验证.

在第一部分中我们描述了 DEMD 的框架和方向确定的算法; 然后在第二部 分给出了本文方法中所用到的 2D Hilbert 变换与特征提取的方法. 第三部分中我 们对 DEMD 的视觉意义进行了讨论. 使用 DEMD 特征进行纹理分割的实验和结 果在第四部分中给出. 第五部分是文章的结论和未来的工作. 


\section{1 方向 EMD 分解(Directional EMD, DEMD)}

\subsection{DEMD 定义}

1998 年Huang等人 ${ }^{[15]}$ 提出EMD方法以进行非平稳、非线性信号处理. 这种工 具首先将信号分解为满足以下条件的成分 $(*)$ : (i ) 零点数目与极值点数目相同 或至多相差 1;（ii）函数关于局部平均对称. 满足这些条件的函数称为Intrinsic Mode Function (IMF). 而进行这种分解的算法就是Huang等人提出的“筛”法. 关 于信号 $x(t)$ 的EMD分解可以形式的表示为下式:

$$
x(t)=\sum_{i=1}^{n} i m f_{i}(t)+r_{n}(t)
$$

其中, $\operatorname{imf}_{i}(t)$ 就是所得的IMF, 而 $r_{n}(t)$ 是单调的残差函数.

EMD 对于处理非平稳信号的优势在于: (1) 对信号的分解是数据驱动的和自 适应的，对于大量实际信号的实验说明这种分解许多情况下是符合物理意义的， 可以得到基本的近似周期成分; (2) 分解的成分(IMF)中可定义合理的瞬时频率特 征(Hilbert 谱); (3) 得到的 IMF 具有波内调制特性(Intra-wave modulation), 把本来 只能由弥散的 Fourier 频率表达的同一成分的信息浓缩到一个成分内部(这正是 Huang 所强调的). 由于这些原因, 自 1998 年 EMD 被提出以来, 其在应用与研究 领域都获得广泛关注. EMD 在信号处理领域应用主要包括: 潮汐、地震、天气、 医学、语音等信号处理, 能够完成将信号分解为不同(缓变)周期成分之和, 信号预 测, 突变检测等的任务; 对于这个工具的研究主要有: 边界处理, 使用不同插值 方法构造包络(如用 B 样条), 终止条件确定(如用双阈值), 对 IMF 进行数学建模 (如用常微分方程模型), EMD 分解的滤波性质(如对于随机分形信号具有半带滤 波性质)等. 与在一维信号处理中所获得的广泛关注相比, 二维 EMD 分解的研究 与应用还刚刚起步，仅有对于使用径向基函数或平面剖分构造二维包络从而进 行二维分解的讨论和对于纹理抽取的应用. 本文提出: 正如 EMD 方法在处理一 维非平稳信号具有优势一样, 其在二维非平稳信号(如多纹理图像)处理方面也有 独特之处. 图 1(a) (c)显示了 EMD 区分纹理的能力.

与一维 IMF 和 EMD 的定义类似, 我们定义二维 IMF 和 DEMD 如下:

定义 1 给定角度 $\theta \in\left[0, \frac{\pi}{2}\right)$, 信号 $u(x, y)$ 若满足下列条件则被称为对应于方 向 $\theta$ 的二维 IMF:

对 $\forall c \in R, v_{1, c}^{\theta}(x)=u(x,[\tan \theta] x+c)(i=1,2), 0 \leq \theta<\frac{\pi}{2}$, 
$v_{2, c}^{\theta}(x)=\left\{\begin{array}{cc}u\left(x,\left[\tan \left(\theta+\frac{\pi}{2}\right)\right] x+c\right), & 0<\theta<\frac{\pi}{2}, \\ u(\cdot, x), & \theta=0,\end{array}\right.$
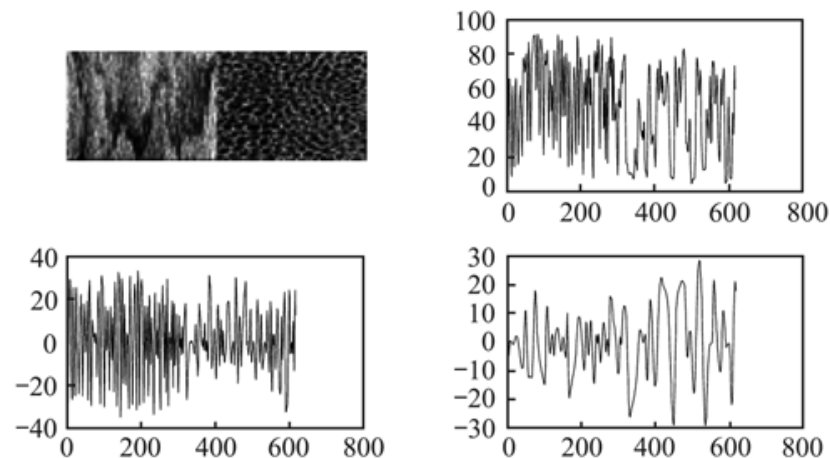

(a)
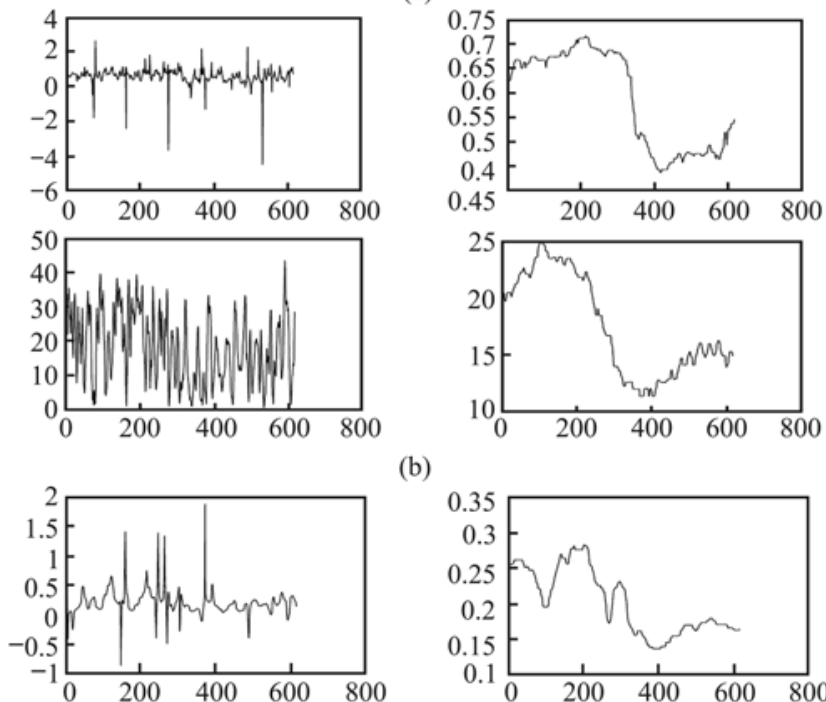

(b)
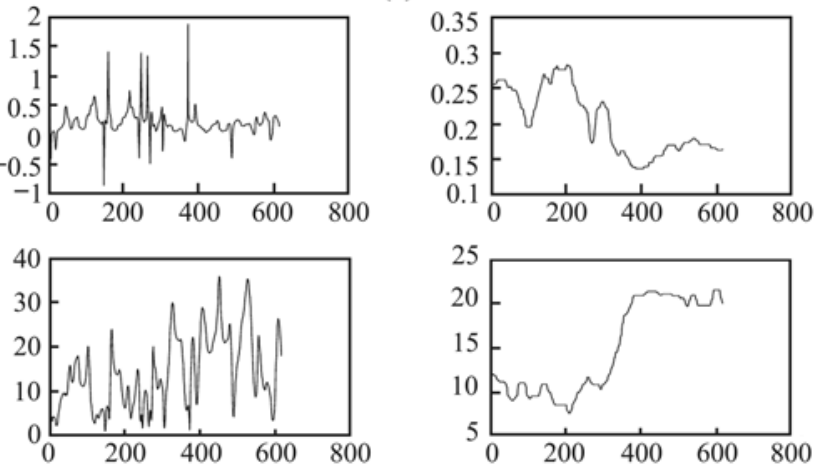

(c)

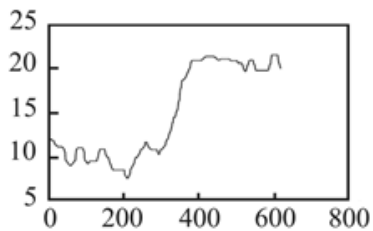

图 1

(a) 依次为一幅自然纹理, 抽取的一行信号, IMF1、IMF2. (顺序: 左上、右上、左下、右下, 下同); (b) IMF1 包络及滤波结果, IMF2 包络及滤波结果; (c) IMF1 瞬时频率及滤波结果, IMF2 瞬时频率及滤波结果

有 $v_{1, c}^{\theta}(x)$ 和 $v_{2, c}^{\theta}(x)$ 都满足条件 $(*) . v_{1, c}^{\theta}(x)$ 和 $v_{2, c}^{\theta}(x)$ 称为 IMF 的一维采样.

定义 2 图像 $f(x, y)$ 对应于方向 $\theta$ 的 DEMD 就是如下分解: 


$$
f(x, y)=\sum_{i=1}^{N} i m f_{i}^{\theta}(x, y)+r_{N}^{\theta}(x, y),
$$

其中, $i m f_{i}^{\theta}(x, y)$ 是对应于方向 $\theta$ 的二维 IMF, 而 $r_{N}^{\theta}(x, y)$ 对于 $v_{1, c}^{\theta}(x)$ 和 $v_{2, c}^{\theta}(x)$ 至 少存在一个单调一维采样. 进行这种分解的算法在下节给出.

\subsection{DEMD 分解算法}

正如得到一维 IMF 的“篮”法一样, 在这里我们给出得到二维 IMF 的二维“篮” 法. 算法如下:

1. 初始化: $r_{i-1}(x, y)=f^{\theta}(x, y)(i=1)$, 其中 $f^{\theta}(x, y)$ 是 $f(x, y)$ 顺时针旋转 $\theta$ 得 到的.

2. 抽取 $\operatorname{imf}_{i}(x, y)$ 第i个IMF:

(i ) 初始化: $h_{0}(x, y)=r_{i-1}(x, y), j=1$;

(ii) 得到 $h_{j-1}(x, y)$ 的平均包络:

$<1>$ 抽取 $h_{j-1}(x, y)$ 每一行的极大、极小值. 然后对这些极大、极小值进行三 次样条插值得到这些一维采样的上下包络 $h_{\text {midupper }}(x, y), h_{\text {midlower }}(x, y)$;

$<2>$ 计算平均包络 $m_{\text {mid }}(x, y)=\left(h_{\text {midupper }}(x, y)+h_{\text {midlower }}(x, y)\right) / 2$;

$<3>$ 通过对 $m_{\text {mid }}(x, y)$ 每列提取极值点和插值得到上下包络 $h_{\text {upper }}(x, y), h_{\text {lower }}(x, y)$. 从而得到 $m_{\text {mid }}(x, y)$ 平均包络 $m_{j-1}(x, y)=\left(h_{\text {upper }}(x, y)+\right.$ $\left.h_{\text {lower }}(x, y)\right) / 2$;

(iii) $h_{j}(x, y)=h_{j-1}(x, y)-m_{j-1}(x, y)$, 令 $j=j+1$;

(iv) 一旦终止条件 $\left(S D:=\sum_{x=0}^{X} \sum_{y=0}^{Y}\left[\frac{\left|h_{1(k-1)}(x, y)-h_{1 k}(x, y)\right|^{2}}{h_{1(k-1)}^{2}(x, y)}\right]<r\right), \quad(\mathrm{r}$ 是所取参数) 满足或 $\mathrm{SD}$ 开始增大, 令 $i m f_{i}(x, y)=h_{j}(x, y)$. 否则令 $j=j+1$. 再转到(ii).

3. $r_{i}(x, y)=r_{i-1}(x, y)-i m f_{i}(x, y)$.

4. 若存在 $r_{i}(x, y)$ 的沿水平、坚直方向的单调一维采样过程就终止. 否则令 $I$ $=i+1$ 转向 2 .

5. 将imf $i, j=1, \cdots, N(N=i)$ 与 $r_{N}(x, y)$ 逆时针旋转 $\theta$ 以得到 $i m f_{j}^{\theta}, j=1, \cdots, N(N=$ i) 和 $r_{N}^{\theta}(x, y)$.

这样对给定的任意二维信号 $f(x, y)$ 都可被分解为如(2)式中的形式. 图 2 显示 了DEMD对自然纹理图像(Brodatz集D $101^{[23]}$ )的分解结果：第一行是自然纹理，使 用DMED水平方向分解的前两个IMF和残差. 第二行是沿 $45^{\circ}$ 方向分解的前两个 $\mathrm{IMF}$ 和残差. 与基于径向基函数的二维 $\mathrm{EMD}^{[5]}$ 方法相比, DEMD不但在速度上具 有优势, 并且由于其主要是在两个方向上力求局部窄带特性, 故在这两个方向上 
可以提取有效的图像特征(方向局部频率).

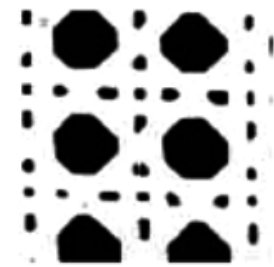

(a)

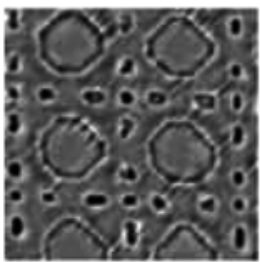

(e)

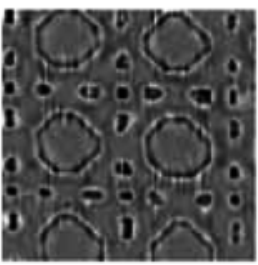

(b)

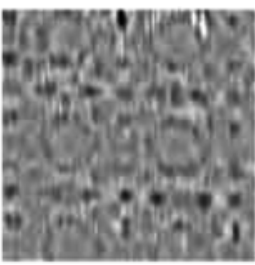

(c)

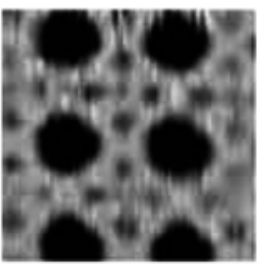

(d)

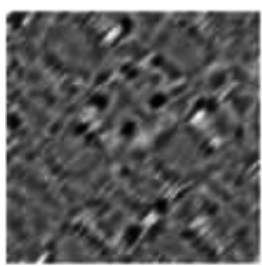

(f)

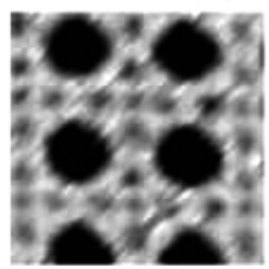

(g)

图 2

(a) 自然纹理D101(Brodatz集 ${ }^{[23]}$ ); (b) (d) D101 沿水平方向进行DEMD分解得到的前两个IMF和残差; (e) (g) D101 沿 $45^{\circ}$ 方向进行DEMD分解得到的前两个IMF和残差

\subsection{DEMD 中方向的确定}

从定义 1,2 中可看出DEMD的关键之一是分解方向的确定. 由纹理的Wold分 解 ${ }^{[18]}$ 可知: 二维规则一致随机场可以唯一的分解为三个正交的部分: 纯随机、规 则随机场 $w(x, y)$; 对应于周期成分的半平面确定性随机场 $p(x, y)$; 对应于方向性 成分的广义瞬时随机场 $g(x, y) . p(x, y)$ 和 $g(x, y)$ 的谱分布函数分别是二维和一维奇 异函数, 而 $w(x, y)$ 的谱分布函数是绝对连续的. 根据这些, 多纹理图像的谱分布 函数包含多个二维和一维奇异函数, 这些都体现了纹理的方向性信息. 为了提高 分类效果我们提取了多个方向, 每一方向对应一个DEMD分解.

在这些分解中, 沿对应于 $g(x, y)$ 成分的方向进行分解是处理沿这些方向的非 平稳性; 而沿对应于 $p(x, y)$ 成分的方向是为了将周期成分与其他部分区分开来. 在提取对应周期成分的二维奇异谱之前, 先提取对应方向成分的一维奇异谱, 这 是通过提取谱的 Radon 变换极大值得到的. 具体算法叙述如下:

1. 多纹理图像 $f(x, y)$ (如图 3(a)) 的谱分布函数 $F(u, v)$ (如图 3(b)).

2. 得到 $F(u, v)$ Radon变换超过门限 $C_{1}$ 的局部极大值点(如图 3(c)) $P_{k}, k=1, \cdots$, $K$, 将它们对应的方向表示为 $\alpha_{j}, j=1, \cdots, K$;

3. 将 $P$ 点周围的值去除作逆Radon变换以得到 $F^{\prime}(u, v)$ (如图 3(d)). 所有其周 围能量和超过 $C_{2}$ 的点按能量排序, 对应方向表示为 $\alpha_{j}(j=K+1, \cdots, K+M)$.

上述过程中 $C_{1}, C_{2}$ 是事先取定的常数. 于是可以对方向 $\alpha_{j}(j=1, \cdots, K+M)$ 进行 
DEMD分解. 根据应用我们可以进行多个DEMD分解.

对步骤 2, 图像经 Radon 变换后, 得到图像: 坚直坐标为角度(实验中取为 $0^{\circ} \sim 180^{\circ}, 5^{\circ}$ 为间隔), 水平坐标为在对应方向上投影位置. 从图 3(c)可看出极大 值点(中心被赋为零位置处)对应角度为 $90^{\circ}$.

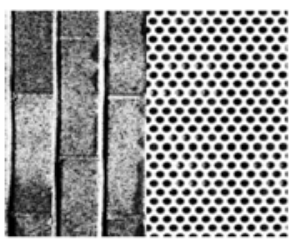

(a)

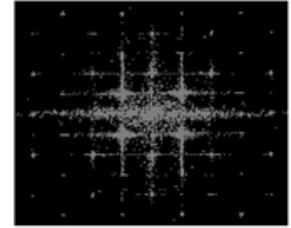

(b)

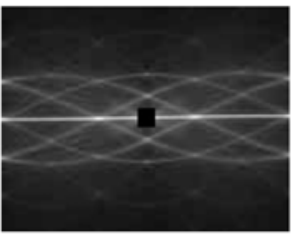

(c)

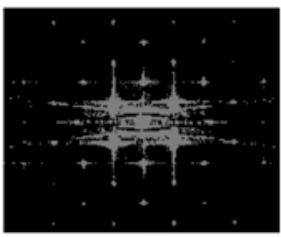

(d)

图 3

(a) 多纹理图像 $f(x, y)$; (b) 谱分布函数 $F(u, v) ;(c) F(u, v)$ Radon 变换全局极大值点;

(d) 瞬时成分去除后的频谱 $F^{\prime}(u, v)$

\section{2 特征提取}

在上部分中我们提出DEMD的定义和算法, 本部分给出了从DEMD中提取特 征的方法. 从 需要首先得到由Hilbert变换定义的复信号. 所以我们先定义信号的二维Hilbert变 换以得到二维信号对应的复信号. 二维Hilbert变换的定义不唯一, 为体现方向 EMD分解的方向性，我们采用二维方向Hilbert变换 ${ }^{[20]}$.

定义 $3 f(x, y)$ 的 Fourier 变换表示为 $F(u, v)$, 则 $f(x, y)$ 对应复信号的 Fourier 变换为:

$$
F_{A}(u, v)=\left\{\begin{array}{cc}
2 F(w), & w \cdot e>0 \\
F(w), & w \cdot e=0 \\
0, & w \cdot e<0
\end{array}\right\}
$$

其中, $\boldsymbol{e}$ 是对应于方向 $\theta$ 的单位向量, $\boldsymbol{w}$ 是坐标向量. $F_{A}(u, v)$ 逆Fourier变换 $f_{A}(x, y)$ 称 为原信号对应于方向 $\theta$ 的复信号. 从而 $f_{A}(x, y)$ 的虚部就是 $f(x, y)$ 对应于方向 $\theta$ 的 Hilbert变换.

参照上述定义, 下面是从 DEMD 分解中提取特征的方法:

令 $\alpha_{j}(j=1, \cdots, K+M)$ 为第三节中算法确定的方向, 根据方向 $\alpha_{j}$ 可以进行 $K+M$ 个DEMD分解.

$$
f(x, y)=\sum_{i=1}^{K+M} i m f_{i}^{j}(x, y)+r_{n}^{j}(x, y), j=1, \cdots, N \quad \text { 其中 } i m f_{i}^{j}(x, y) \text { 表示对应于方 }
$$
向 $\alpha_{j}$ 的DEMD分解的第 $i^{\text {th }}$ 个IMF.

令 $i m f_{i H}^{j}(x, y)$ 为 $i m f_{i}^{j}(x, y)$ 对应于方向 $\alpha_{j}$ 的 2-D Hilbert变换, $f_{i A}^{j}(x, y)$ 是对应 
的分析函数.

下面定义特征:

$$
A_{i}^{j}=\left[\left(i m f_{i}^{j}\right)^{2}+\left(i m f_{i H}^{j}\right)^{2}\right]^{\frac{1}{2}} \text { 和 } \theta_{i}^{j}=\arctan \left(\frac{i m f_{i H}^{j}}{i m f_{i}^{j}}\right), i=1, \cdots, K+M, j=1, \cdots, N .
$$

这样就得到第 $j^{\text {th }}$ DEMD的第 $i^{\text {th }}$ IMF的频率. 这里频率是通过分别沿 $\alpha_{j}$ 和 $\alpha_{j}+$ $90^{\circ}$ 的方向导数 $\frac{d}{d j^{k}}$ 得到

$$
\omega_{i}^{j_{k}}=\frac{d}{d j^{k}} \theta_{i}^{j}=\frac{i m f_{i}^{j} \cdot \frac{d}{\vec{d}_{j}{ }^{k}} i m f_{i H}^{j}-\frac{d}{\vec{d}_{j}{ }^{k}} i m f_{i}{ }^{j} \cdot i m f_{i H}^{j}}{\left(i m f_{i}^{j}\right)^{2}+\left(i m f_{i H}^{j}\right)^{2}}, i=1, \ldots, K+M, j=1, \cdots, N \text { 和 }
$$

$k=1,2$.

其中, $k=1$ 对应 $\alpha_{j}$ 方向, $k=2$ 对应 $\alpha_{j}+90^{\circ}$ 方向.

通过上述过程我们得到对应于每一个像素的三个特征:

$$
V(m, n)=A_{i}^{j}(m, n), \omega_{i}^{j_{k}}(m, n)_{1 \leq i \leq M, 1 \leq j \leq N, k=1,2 .}
$$

\section{DEMD 特征区分纹理的视觉意义}

关于人视觉上区分纹理的机理已经有许多深入的研究, 如认为原纹理的二 阶或更高阶统计量对区分纹理是足够的，或认为纹理基元(texton)及基元间的统 计信息才是正确的特征 ${ }^{[21]}$, 近年来又有人将纹理区分与多尺度、多方向滤波器的

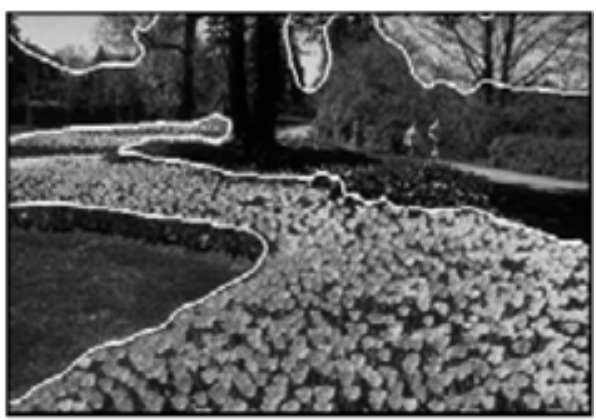

图 4 一幅由多种尺度纹理组成的自然图像

输出结合起来，认为使用小波或 Gabor滤波器输出的幅值或其他特征 进行纹理区分符合人视觉区分纹理 的机理 ${ }^{[22]}$. 本文认为: 人视觉上区分 纹理是根据各种纹理相对的最高频、 次高频等进行分辨，并且这种频带的 区分是自适应的. 如图IV所示为多个 尺度纹理组成的自然图像. 人视觉上 很容易的将花地与草地区分开, 但并 不是像Gabor滤波器方法和小波方法 那样比较不同图像部分对某个尺度/ 频率的能量以进行区分，而是在草地部分自然的取小尺度为优先考虑的局部大 尺度，花地部分则相反，因而人视觉上区分自然图像中不同纹理是根据局部图像 的最高频、次高频等进行处理. 本文的算法和下面的实验说明, EMD方法反映了 人视觉的这种现象：EMD分解的过程反映了提取局部高频、次高频等的过程，而 
提取Hilbert谱反映了这种局部频率的计算和识别. 下节的实验说明了这个新框架 的有效性.

\section{4 实验及结果}

这一节我们将描述使用DEMD进行纹理分割的实验验证. 所用图像为来自 标准纹理库Brodatz集 ${ }^{[23]}$ 中纹理组成的合成图像. 对每个图像进行两组DEMD分 解 $(K=1, M=1)$, 每个分解采用两个IMF的特征, 对每个IMF提取两个频率特征和 一个包络特征, 再加上两个坐标特征, 然后使用 14 个特征进行 $k$ 均值聚类分割. (使用两个方向进行分解的原因是增加一个方向的分解意味着增加 6 个特征, 过 多特征会增加计算量, 并且使聚类容易陷入局部极小值和发生不收玫的情况. 另 一方面, 在本文进行的各实验中继续增加方向数目并不能明显改善实验效果. )最 后, 使用形态学膨胀、腐蚀 ${ }^{[20]}$ 进行分割结果优化.

考虑 Brodatz 集中纹理组成的合成图像. 分别将 Brodatz 集中的纹理组成两幅 测试图像. 图 5(a) (d)表示了由三种纹理组成的 200×200 图像的分割结果, 误分 率为 $5 \%$. 由于我们考虑了坐标特征，在中间纹理的狭长部分有一定误差. 由

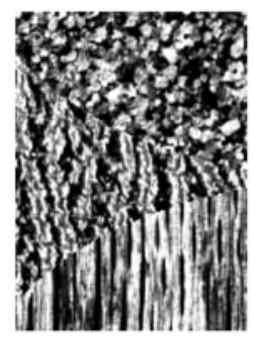

(a)

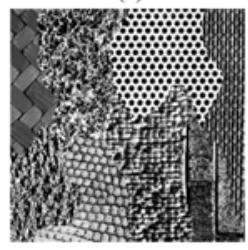

(e)

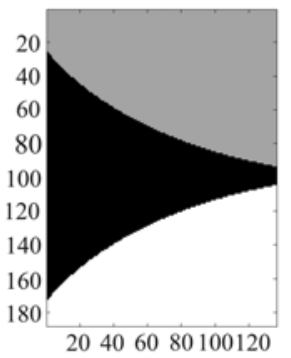

(b)

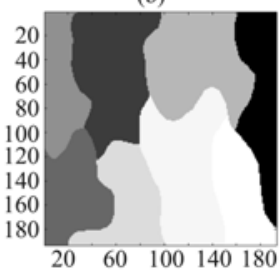

(f)

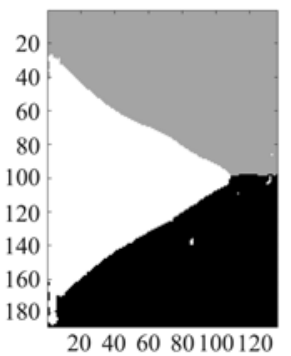

(c)

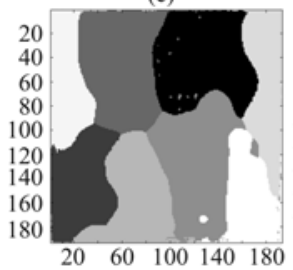

(g)

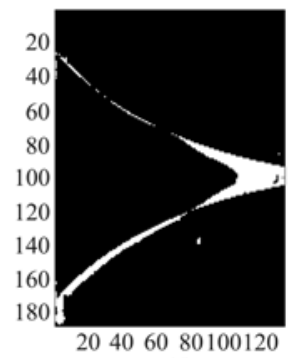

(d)

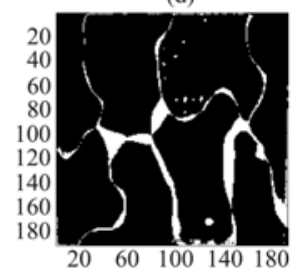

(h)

图 5

(a)、 (e) 组合纹理和自然图像; (b)、(f) 组合模板; (c)、(g) 分割结果; (d)、(h) 分割误差

Brodatz 集中八种纹理组成的合成图像分割结果在图 5(e) (h)中给出. 由于包含大 尺度和较难区分的纹理误分率增为 $9 \%$.

\section{5 结论}


本文中我们提出一种新的空频分析方法—DEMD, 并将之用于纹理分割. 与小波和 Gabor 滤波器方法这些传统空频分析方法相比, DEMD 的优点在于: 它 是完全数据驱动的自适应分解, 不像小波那样只能反映图像在某个函数平移、伸 缩形成的基上的能量, 也不像 Gabor 滤波器那样有几余性(实际上 DEMD 是近似 的正交分解). 作为适合处理一维非平稳信号的 EMD 方法的二维推广, DEMD 具 有两个优点: 方向性和能够提出有效特征. 实验验证了 DEMD 方法对于纹理分割 的有效性. 本文还给出了 DEMD区分纹理的视觉解释. 虽然这种方法现在在分割 效果上还不能超过现知的最好方法, 但其基于全新的多尺度分解结构, 具有完全 数据驱动的自适应性, 并且反映独特的视觉意义, 为纹理分析打开了新的方向.

下一步的工作包括提高 DEMD 分割效果和将之用于其他纹理处理问题如纹 理检索.

\section{参考文献}

1 Perona P, Malik J. Scale-space and edge detection using anisotropic diffusion. IEEE Trans Pattern Analysis and Machine Intelligence, 1990, 12(7): 629 639. [DOI]

2 Goutsias J, Heijmans H J A M. Nonlinear Multiresolution Signal Decomposition Schemes_—Part I: Morphological Pyramids. IEEE Trans Image Processing,2000, 9(11): 1862 1876. [DOI]

3 Burt P, Adelson E. The Laplacian Pyramid as a Compact Image Code. IEEE Trans on Communications, 1983,31(4): $532 \sim 540$ [DOI]

4 Mallat S G. A theory for multiresolution signal decomposition: The wavelet representation. IEEE Trans Pattern Analysis and Machine Intelligence, 1989,11(7): 674 693 [DOI]

5 Nunes J C, Bouaoune Y, Delechelle E, et al. Texture analysis based on the bidimensional empirical mode decomposition. Machine Vision and Application, 2003, Accepted

6 Comer M L, Delp E J. Segmentation of textured images using a multiresolution Gaussian autoregressive model. IEEE Trans on Image Processing, 1999, 8: 408 420 [DOI]

7 Tuceryan M, Jain A K. Texture segmentaiton using Voronoi polygons. IEEE Trans on Pattern Analysis and Machine Intelligence, 1990, 12(2): 211 216 [DOI]

8 Chaudhuri B B, Sarkar N. Texture segmentation using fractal dimension. IEEE Trans on Pattern Analysis and Machine Intelligence, 1995, 17(1): 72 77 [DOI]

9 Jacobson L D, Wechsler H. Joint spatial/spatial-frequency representation. Signal Processing, 1988, 14: $37 \sim 68$ [DOI]

10 Lu C S, Chung P C, Chen C F. Unsupervised texture segmentation via wavelet transform. Pattern Recognition, 1997, 30(5): 729 742 [DOI]

11 Bovik A C, Clark M, Geisler W S. Multichannel texture analysis using localized spatial filters. IEEE Trans on Pattern Analysis and Machine Intelligence, 1990, 12(1): 55 73 [DOI]

12 Weldon T P, Higgins W E, Dunn D F. Efficient Gabor-filter design for texture segmentation. Pattern Recognition, 1996, 29(12): 2005 2016 [DOI]

13 Randen T, Husoy J H. Multichannel filtering for image texture segmentation. Opt Eng, 1994, 33(8): 2617 2625

14 Cohn L. Time-Frequency Analysis. Englewood Cliffs, NJ: Prentice-Hall, 1995

15 Huang N E, Shen Z, Long S R, et al. The empirical mode decomposition and the Hilbert spectrum for nonlinear and non-stationary time series analysis. Proc R Soc Lond A, 1998, 454: 903 995 
16 Yue H Y, Guo H D, Han C M, et al. A SAR interferogram filter based on the empirical mode decompositin method. IGARSS'2001

17 Long S R. Use of the empirical mode decomposition and Hilbert-Huang transform in image analysis, World Multi-conference on Systemics, Cybernetics and Informatics, Cybernetics And Informatics: Concepts And Applications (Part II), 2001

18 Francos J M, Meiri A Z, Porat B. A unified texture modle based on a 2-D Wold-like decomposition. IEEE Trans on Signal Processing, 1993, 41: 2665 2678 [DOI]

19 Liu Z X, Wang H J, Peng S L. Texture segmentation using directional empirical mode decomposition. IEEE ICIP'04, Accepted

20 Bulow Th ,Sommer G. Hypercomplex Signals — A Novel Extension of the Analytic Signal to the Multidimensional Case. IEEE Trans on Signal Processing, 2001, 49(11): 2844 2852 [DOI]

21 Julesz B. Textons, the elements of texture perception, and their interactions. Nature, 1981, 290, 12 March: 91 97 [DOI]

22 Tomita F, Tsuji S.Computer Analysis of Visual Textures, Kluwer Academic, Boston, 1990

23 Brodatz P. Textures-A Photographic Album for Artists and Designers, Dover, New York, 1966 